\title{
L'HOMME L'Homme
}

$179 \mid 2006$

Des raisons du terrain

\section{Choisir un nom de famille...}

Une approche pluridisciplinaire des implications de la réforme du nom

Marie-Claude Casper, Frédérique Granet et Charles-Henry Pradelles de Latour

\section{(2) OpenEdition \\ Journals}

Édition électronique

URL : http://journals.openedition.org/lhomme/24061

DOI : 10.4000//homme.24061

ISSN : 1953-8103

Éditeur

Éditions de l'EHESS

Édition imprimée

Date de publication : 1 septembre 2006

Pagination : 201-217

ISSN : 0439-4216

\section{Référence électronique}

Marie-Claude Casper, Frédérique Granet et Charles-Henry Pradelles de Latour, « Choisir un nom de famille... », L'Homme [En ligne], 179 | 2006, mis en ligne le 01 janvier 2008, consulté le 19 avril 2019. URL : http://journals.openedition.org/lhomme/24061; DOI : 10.4000//homme.24061 


\title{
Choisir un nom de famille... Une approche pluridisciplinaire des implications de la réforme du nom
}

\author{
Marie-Claude Casper \\ Frédérique Granet \\ Charles-Henry Pradelles de Latour
}

\section{$\mathrm{D}$}

ANS LE CADRE D'UNE MUTATION du droit de la famille et réalisant un rapprochement avec certaines législations européennes, une modification sensible du système nominatif en France vient d'être opérée ${ }^{1}$.

Cette législation, qui introduit la possibilité d'un choix là où une transmission était automatique, offre pour l'étude du nom et de l'acte de nomination qui l'instaure, un terrain de recherche privilégié.

De façon plus générale, cette loi pose-t-elle les fondations d'un nouvel ancrage d'un sujet à ce qu'on nomme une "famille " ? Parallèlement, les représentations de ce qu'est une famille dans notre monde occidental ne sont-elles pas en train d'évoluer vers de nouvelles formes d'appartenance familiale?

Le système nominatif reconnu officiellement dans l'ensemble des pays européens distingue deux entités nominatives, à savoir le nom et le prénom. Le nom, jusque-là imposé et désigné par la loi, à la différence du prénom, s'appuie sur une transversalité filiative qui relie une personne avec ses ascendants et ses descendants. Ainsi le nom comme emblème d'une appartenance à cette communauté familiale inscrit la personne qui le porte dans une continuité généalogique. Le prénom, quant à lui, issu d'un choix, accueille de façon éminemment individuelle une personne à sa naissance. Il est porteur de la singularité d'une rencontre entre des parents et un enfant.

1. Lois du 4 mars 2002 et du 18 juin 2003 relatives au nom de famille et à sa dévolution ainsi que le décret du 29 octobre 2004 portant application de ces lois.

Cet article collectif a été rédigé avec la participation de Marie-Lorraine PradellesMonod et Marie-Paule Chevalérias, maittres de conférences en psychologie et psychopathologie cliniques, de Véronique Jaworski, maître de conférences en droit et Louis-Albert Devillairs, attaché temporaire d'enseignement et de recherche en droit. 
Depuis janvier 2005, date de mise en application de la loi, la question $\mathrm{du}$ choix se pose aussi pour l'attribution du nom. Selon la nouvelle législation, le nom dépend, en plus de l'établissement d'une filiation, d'une volonté individuelle des parents. À la naissance d'un premier enfant, là où le nom était jusque-là imposé par la loi, il est désormais possible de choisir entre le nom du père, celui de la mère, ou les deux noms accolés, ce qui entraîne une transformation importante des conditions de sa transmission.

Quelles hypothèses pouvons-nous formuler en ce qui concerne les incidences de ces modifications sur le rapport intime du sujet à son nom ? La nouvelle donne, tout à la fois juridique et sociale, affecte-t-elle le nom dans sa valeur éminemment symbolique de pur représentant? Comment le lien entre nom et transmission s'en trouve-t-il éclairé ?

Ce sont autant d'interrogations auxquelles cet article tente de répondre selon une approche pluridisciplinaire ${ }^{2}$ qui associe les sciences juridiques, anthropologiques et psychologiques.

Seront tout d'abord soulignés les changements juridiques qui accompagnent la notion de "choix " et ce qui en découle (la disparition de ce nom que l'on appelle patronymique), ce qui nous permettra ensuite d'en explorer les possibles incidences sur le versant social et sur le versant subjectif.

Cette organisation qui, en Occident, associe nom et prénom pour constituer l'identité nominative d'une personne n'est cependant pas transposable dans toutes les sociétés. En effet, les systèmes de nomination que mettent au jour les études anthropologiques sont soutenus par d'autres formes d'organisation où les catégories du nom et du prénom n'ont pas cours, où les termes nominatifs expriment autrement l'idée d'un choix et les distinctions entre le collectif et l'individuel.

L'approche psychologique inscrit ce choix dans une histoire familiale. Comment cette décision, dans une parole, sera-t-elle liée à une histoire aux multiples ramifications (histoire du père, de la mère, histoire du couple...) et quels en sont les motifs à la fois explicites et implicites? À partir d'entretiens de recherche et des données issues d'une pratique clinique de psychologue auprès d'enfants et d'adultes, nous développerons quelques-unes des pistes de réflexion qu'ouvre cette donne juridique en ce qui concerne les liens d'un sujet à sa famille.

2. Intitulé «La transmission du nom de famille en France et sa nouvelle donne juridique : fonctions sociales et enjeux psychologiques du nom ", ce programme interdisciplinaire du CNRS sur "Les systèmes de dénomination : origine, évolution, diversité, devenir » est dirigé par CharlesHenry Pradelles de Latour, UMR 8556 CNRS. 


\section{Du nom patronymique au nom de famille les nouvelles fondations juridiques du nom}

Jusqu'à cette réforme, le Code civil ne contenait aucune disposition relative à l'attribution $\mathrm{du}$ nom ${ }^{3}$. Le fondement du droit français en matière de dévolution du nom reposait en effet sur des règles coutumières, issues d'une tradition féodale organisée sur le modèle de la filiation légitime et de la prééminence du père. Ainsi, dans la famille fondée sur le mariage, l'homme seul avait jusqu'à présent le droit de transmettre son nom à sa descendance; la femme, quant à elle, était socialement identifiée par l'accouchement. Depuis un siècle, des modifications juridiques importantes - l'abolition du droit de punition en 1895, de la puissance matrimoniale en 1938 et l'introduction en 1970 de l'autorité parentale partagée ont retiré au père ses prérogatives. Ces lois affectent le père et le groupe familial avec lequel il est lié.

Aujourd'hui, sous l'impulsion d'un courant égalitaire entre père et mère et des sources européennes du droit, les lois du 4 mars 2002 et du 18 juin $2003^{4}$ modifient de manière essentielle les règles d'attribution du nom qui devient très officiellement "nom de famille ", expression substituée à celle de «nom patronymique ». Le nom de la mère comme celui du père devient transmissible. À cette fin est introduit le principe d'un choix ${ }^{5}$, pierre angulaire de ce nouveau dispositif. En effet, la loi qui a opté pour une attribution qui n'est plus impérativement fixée mais encadrée, laisse aux parents une liberté de choix dans la limite des noms que les conditions d'établissement de la filiation pour l'enfant rendent disponibles. Le nom de famille sera ainsi constitué d'un ou de deux noms parmi ceux des parents. Valable pour tous les enfants d'un couple, ce choix ne peut être exercé qu'une seule fois ${ }^{6}$ à l'occasion de la naissance du premier d'entre eux ${ }^{7}$. Une mesure transitoire

3. Certes, la loi du 6 fructidor an II disposait qu' «aucun citoyen ne pourra porter de nom ni de prénom autres que ceux exprimés dans son acte de naissance». Le législateur était intervenu à plusieurs reprises par la loi du 3 janvier 1972 relative au nom de l'enfant naturel, celle du 23 décembre 1985 autorisant l'usage des noms paternel et maternel et celle du 8 janvier 1993 sur le changement de nom, mais rien n'était prévu en ce qui concernait sa dévolution.

4. Loi $n^{\circ} 2002-304$ du 4 mars 2002 relative au nom de famille modifiée par la loi $n^{\circ} 2003-516$ du 18 juin 2003 relative à la dévolution du nom de famille ainsi que le décret $n^{\circ} 2004-1159$ du 29 octobre 2004 portant application de la loi n 2002-304 du 4 mars 2002 modifiée relative au nom de famille et modifiant diverses dispositions relatives à l'état civil

5. Article 311-21 du Code civil, inscrit dans une section V nouvelle intitulée «Des règles de dévolution du nom de famille». Elle est ajoutée au chapitre $1^{\text {er }}$ du titre VII du livre I $^{\text {er }}$ du Code, consacré aux dispositions communes applicables aux enfants légitimes et aux enfants naturels.

6. Articles 311-21 et 334-2.

7. Article 311-23. 
énoncée dans le décret d'application ${ }^{8}$ permet aux parents qui exercent en commun l'autorité parentale de demander conjointement pour l'aîné des enfants, s'il a moins de treize ans au $1^{\text {er }}$ septembre 2003 ou à la date de la demande, l'adjonction en deuxième position du nom de famille du parent qui n'a pas transmis le sien. Ce double nom est dévolu à l'ensemble de leurs enfants communs nés ou à naître.

L'attribution du nom est fonction du mode de filiation établi pour l'enfant, à savoir : une filiation légitime, une filiation naturelle accompagnée ou non d'une légitimation, ou une filiation adoptive (que nous n'aborderons pas).

Jusqu'au $1^{\text {er }}$ janvier 2005, selon une logique patriarcale de la famille, l'enfant légitime, conçu ou né dans le mariage et présumé avoir pour père le mari de sa mère, acquérait de plein droit le nom de celui-ci ${ }^{9}$. Depuis, lorsque la filiation est établie à l'égard des deux parents, les époux ont la possibilité, par déclaration conjointe, de choisir le nom de famille entre celui du père, celui de la mère, ou l'ordre de leur accolement dans la limite d'un nom de famille pour chacun d'eux. À défaut de déclaration conjointe à l'officier de l'état civil, notamment en cas de désaccord, l'enfant acquiert le nom du mari. Lorsque les époux ou l'un des époux portent un double nom de famille, ils peuvent, par déclaration écrite conjointe, ne transmettre qu'un seul nom à leurs enfants.

Pour un enfant né hors mariage, l'attribution du nom dépend de l'établissement de sa filiation, qui, lorsqu'elle est naturelle, est divisible. Celleci peut être établie exclusivement à l'égard de la mère, exclusivement à l'égard du père, ou encore à l'égard des deux parents simultanément ou successivement. Aussi l'enfant naturel acquiert-il le nom de celui de ses parents à l'égard duquel sa filiation est établie en premier lieu ${ }^{10}$. En cas de simultanéité, les modalités d'attribution du nom sont identiques à celles qui prévalent pour l'enfant légitime. En cas de filiation unique, l'enfant prend le nom de son seul parent légal. Lorsque la filiation est établie successivement, le nom de l'enfant est celui du parent à l'égard duquel elle est établie en premier lieu ${ }^{11}$ avec une possibilité, sur déclaration conjointe,

8. Article 18 du décret $n^{\circ} 2004-1159$ du 29 octobre 2004 portant application de la loi $\mathrm{n}^{\circ} 2002$ $304 \mathrm{du} 4$ mars 2002 modifiée relative au nom de famille et modifiant diverses dispositions relatives à l'état civil.

9. La loi $\mathrm{n}^{\circ} 85-1372$ du 23 décembre 1985 relative à l'égalité des époux dans les régimes matri-

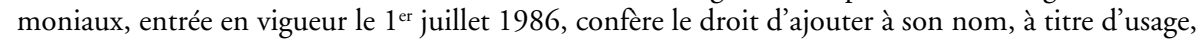
celui de ses parents qui ne lui a pas transmis le sien (article 43 alinéa 1). Pour les enfants mineurs, cette faculté est mise en œuvre par les titulaires de l'autorité parentale. L'enfant devenu majeur ou émancipé peut à tout moment mettre fin à cet usage.

10. Article 334-1 du Code civil.

11. Article 334-1 du Code civil. 
de demander un changement de nom, et cela suivant les mêmes conditions que pour une naissance légitime ${ }^{12}$.

Dans les sociétés occidentales le(s) prénom(s) et le(s) nom(s) enregistrés par l'état civil sont nettement différenciés par leurs formations (même si certains prénoms ont pris dans l'histoire valeur de nom), par leurs usages contrastés (familier ou officiel) et par leur mode d'acquisition, puisque jusqu'à présent seuls les prénoms faisaient l'objet d'un choix. Cette opposition entre prénom et nom renvoie à une distinction que nous pouvons faire entre nom individuel et nom collectif.

Qu'en est-il de cette distinction dans des sociétés traditionnelles, africaines par exemple, où la culture est, à la différence de la nôtre, tributaire des lois de la communication orale, où le système nominatif n'est pas figé par son enregistrement écrit et sa régulation légale?

\section{Nom collectif et nom individuel une approche anthropologique}

Pour illustrer notre propos, commençons par examiner les conditions de choix et d'attribution des noms de personnes chez les Dogons du Mali, pour voir ensuite comment les noms individuels et collectifs sont généralement formés et utilisés dans les sociétés de l'Afrique noire.

Quelques jours après sa naissance, l'enfant dogon reçoit de son père un nom personnel ordinaire, appelé boy toy, "nom semence ", qui peut être prédéterminé ou choisi (Lifchitz \& Paulme 1952). Certains de ces noms répondent en effet à la numération ordinale des naissances: Atié, "le premier ; Antimmu, «le second »; Atandou, «le troisième », etc. D'autres sont choisis pour avoir été portés par des grands-parents décédés, ou sont retenus pour leur signification particulière: Ambara, "Que dieu croisse "; Amana, "Que les hommes restent unis ». Quelques semaines après leur naissance, les garçons et les filles sont présentés au prêtre totémique de leur lignage qui leur confere un nom secret, boy dama, lequel n'est utilisé qu'entre le prêtre et l'enfant. Ce nom est confidentiel car il ancre et fonde l'existence de tout être humain dans l'au-delà de la vie sociale. Enfin, lorsqu'un nouveau-né est apporté pour la première fois dans la famille de sa mère, il y reçoit un nom appelé, na boy, "le nom de la famille de la mère ", qui est construit de la même manière que les deux noms précédents. En sus de ces trois premiers noms de base, les Dogons ont un nom collectif, ginu, qui les relie au lignage de leur père: Atandou Tahad est «Atandou de la famille Tahad", Amabar Ambata, "Amabara de la famille Ambata ». 
Lorsque les garçons devenus préadolescents sont circoncis et initiés, leur père leur révèle la devise, tige, de l'ancêtre dont ils ont hérité l'âme, kikinu, à leur naissance (Ganay 1959). Plusieurs garçons peuvent partager le même protecteur et avoir ainsi le même tige, qui se substitue progressivement à leur nom ordinaire, car ces devises mettent en valeur leurs caractéristiques honorifiques, héritées du passé. "La bière du chemin " est un tige qui rappelle par exemple que l'individu félicité est généreux car, sur le chemin, il offre de la bière de mil aux passants. Ces devises ne sont pas propres aux individus, il en existe qui se rapportent aux lignages, aux localités célèbres ou aux castes comme celles des forgerons ou des griots. Ces noms d'éloges peuvent être ajoutés les uns à la suite des autres pour remercier, flatter ou honorer un homme ayant atteint la maturité. Les femmes, qui n'ont pas de tige, prennent pour leur part le nom de l'association dans laquelle elles entrent à la suite de leur excision.

De façon générale, l'anthroponymie des sociétés africaines, à l'instar de celle des Dogons, est plurielle et mouvante : tout individu reçoit à la naissance plusieurs noms et en change au cours de son existence. Les noms portés par les personnes au cours de leur vie témoignent non seulement des différentes identifications constitutives d'une personnalité plurielle, mais aussi et surtout des différentes sphères relationnelles - lignages des deux parents, monde des ancêtres et des totems, associations et rangs honorifiques auxquelles tout individu appartient simultanément et successivement.

Une distinction entre nom individuel et nom collectif s'opère dans toutes les sociétés africaines à l'exception toutefois des Bochimans du désert du Kalahari qui, n'ayant pas de groupe de filiation, se constituaient en cercles d'homonymes (Fabian 1965). Selon cette pratique connue sous l'appellation de "système !kung", tous les porteurs du même nom individuel formaient un groupe exogamique de solidarité dont les fonctions étaient proches de celles du lignage ou du clan. Quant aux Pygmées, qui n’avaient pas non plus de groupe de filiation, ils se désignaient par des noms de camp. Toutes les sociétés africaines ont donc, d'une façon ou d'une autre, des noms individuels faisant l'objet d'un grand choix et des noms collectifs non choisis, mais leurs usages respectifs different assez radicalement des nôtres.

Les noms individuels se répartissent en trois catégories : ceux qui sont prédéterminés par le type de naissance de l'enfant, ceux qui sont préformés comme nos prénoms et ceux qui sont créés au moment de la naissance à partir de noms communs.

Dans la première catégorie, le nom n'est pas choisi mais attribué en fonction d'une donnée précise concernant certaines caractéristiques de l'arrivée au monde de l'enfant. On y trouve les noms numériques qui, comme chez les Dogons, classent les individus par leur ordre de naissance, 
et les noms calendaires. Ces derniers répartissent les individus en fonction de repères temporels qui marquent la naissance, tel le jour de la semaine, par exemple «Né le dimanche » comme chez les Ashanti, ou le moment de la journée comme chez les Luo d'Ouganda: "Né le matin», "Né à midi ", "Né dans la nuit». Dans cette catégorie on trouve également des noms prédéterminés par la spécificité de la naissance de l'enfant; c'est le cas des jumeaux et du frère ou de la sœur qui les suit, lesquels reçoivent des noms génériques qu'ils sont seuls à pouvoir porter. Dans certaines sociétés, les enfants nés par le siège, ou nés après trois enfants consécutifs de même sexe portent aussi des noms préétablis. Quant aux enfants nés après la mort de leur père ou ceux nés après le décès des enfants les ayant précédés, ils portent soit des noms d'effacement, "Je ne reste nulle part", soit des noms d'inexistence, "Chose jetée ", afin que les esprits maléfiques ayant perturbé leurs prédécesseurs ne fassent pas attention à eux.

Dans la deuxième catégorie des noms individuels, quelques anthroponymes forment des batteries qui appartiennent généralement à des groupes constitués : clans, chefferies ou sociétés. Dans certains clans chez les Soninké du Mali et de Mauritanie, les noms individuels, communs aux sociétés musulmanes des régions subsahariennes, sont choisis par le père pour les deux premiers enfants, et par la mère pour le troisième et le quatrième, et ainsi de suite de deux en deux. On dit généralement que "ces noms sont pris par les parents sur la tête d'un ancêtre, ou sur celle d'un de leurs consanguins ou d'un de leurs amis " qui devient alors pour l'enfant nommé une sorte de parrain plus ou moins marqué selon les sociétés. Le choix du nom peut être aussi fait par une assemblée familiale ou par un devin surtout lorsqu'il s'agit de reprendre le nom d'un défunt. Chez les Darabo, le père égrenait autrefois des noms individuels connus devant le nouveau-né en attendant qu'il éternue. Le nom prononcé juste avant l'éternuement était retenu comme étant celui qui est accepté.

Dans la troisième catégorie des noms individuels, le choix est très ouvert, le nom étant créé par les parents à partir de circonstances qui précèdent ou accompagnent la naissance de l'enfant. Cette catégorie a été largement développée par plusieurs auteurs dont Middleton (1957) et Houis (1963).

$\mathrm{Si}$, donc, dans certaines sociétés africaines, le choix des noms individuels est limité par un stock de noms prédéterminés par un prêtre, un devin ou une assemblée familiale, dans de nombreuses autres sociétés ce choix est très large.

À l'inverse des noms individuels, les noms collectifs africains ne font pas l'objet d'un choix. Ce sont des noms de groupes de filiation constitués, lignages et clans patri- ou matrilinéaires, ou encore des noms de groupes résidentiels désignant une localité. Ces noms sont composés au moyen du 
nom individuel de l'ancêtre fondateur, de celui du héros éponyme ayant présidé à leur fondation, ou encore d'un emblème totémique relevant des règnes animal, végétal ou cosmique. Il arrivait parfois que le surnom d'un ancêtre remplace celui du fondateur, comme chez les Azandé du Soudan, mais lorsque ce surnom était adopté, il restait fixe pendant une ou deux générations en attendant d'être remplacé par le surnom d'un ancêtre plus récent. Les noms collectifs sont utilisés de façon majoritaire comme termes de référence afin de différencier les unités composant l'organisation de base d'une société traditionnelle, mais ils sont aussi très employés comme termes d'adresse pour honorer leurs représentants. Associés à des généalogies prestigieuses, aux hauts faits de leurs fondateurs, aux alliances contractées avec d'autres groupes, aux adjuvants surnaturels qui ont sauvé leurs ancêtres au cours de l'histoire, les noms collectifs, chargés de souvenirs et de tradition, sont glorifiés par les griots de l'Afrique occidentale et honorés par tous les ressortissants d'une société sous la forme de devises courtes ou de titres témoignant d'un rang. Plus les noms collectifs relèvent de groupes de filiation élevés dans la hiérarchie sociale, plus ils conferent à leurs membres de la renommée; plus les individus avancent en âge, plus ils sont salués et honorés par les noms collectifs des nombreux groupes - lignage, caste, association et ethnie - qu'ils ont la charge de représenter et de perpétuer. Ainsi, dans le temps de l'histoire, les noms individuels devenus prestigieux se fossilisent en noms collectifs, alors que dans le temps d'une vie, au fur et à mesure que les personnes vieillissent, l'usage des noms collectifs honorifiques finit par supplanter celui des noms individuels. Entre ces deux types de noms il existe donc une passerelle à double sens.

L'anthropologie des nomenclatures africaines apporte ainsi quelques informations précieuses qui vont nous permettre de différencier les noms individuels des noms collectifs afin de mieux cerner ce qu'implique le changement inauguré par la loi actuelle.

Quelle que soit la nature des noms individuels, prédéterminés, choisis dans un stock de noms préformés ou créés au moment de la naissance, leur dation, officielle ou non, constitue leur fondement initial. En associant indéfectiblement une essence à l'existence d'une personne, la nomination (naming) confere au nom propre son caractère de "désignateur rigide " si bien défini par Kripke (1982). Selon cet auteur, cette suture anthropologique n'est valable que dans un univers particulier de discours où les interlocuteurs doivent, pour communiquer, partager une connaissance commune des noms et des contenus relatifs aux personnes désignées. En Afrique, les noms de chien étant des messages adressés aux voisins à l'instar de certains noms de personne, la différenciation entre ces deux types de noms ne peut être effectuée que par le contexte et les modalités de leur énonciation. 
À la différence des noms individuels, les noms collectifs réferent à des groupes de filiation qui existent en soi, depuis leur fondation, indépendamment des générations qui se renouvellent en leur sein. Ayant ainsi valeur de concept, ils sont souvent eux-mêmes des attributs (totémiques ou historiques) semblables à ceux que véhiculent les surnoms. De même que les lignages et les clans existent en dehors des personnes qui les composent, celles-ci s'identifient au nom de leur groupe de filiation de façon secondaire lorsqu'elles en deviennent des membres actifs - des reproducteurs. Si le nom individuel est fondé à la naissance d'un être humain sur son unicité, le nom collectif est avalisé au cours d'un devenir, lorsqu'un individu avançant en âge associe sa réussite parentale et personnelle à la notoriété de son unité sociale. Le nom collectif est ainsi non seulement une extension honorifique du nom propre individuel, mais aussi et surtout l'ancrage d'un sujet dans une généalogie pour en être, en tant que géniteur ou procréatrice reconnu(e), un chaînon indispensable. C'est ainsi que, dans notre société, le nom collectif associé à une lignée agnatique est devenu un patronyme, le nom du père.

Dans les sociétés de l'écrit et les sociétés orales, la dation et l'usage des noms individuels et ceux des noms collectifs different sensiblement; mais dans les deux univers le choix porte essentiellement sur le nom individuel et jamais sur le nom collectif. Que signifie donc le fait que le nom collectif soit soumis désormais à un choix personnel dans notre société? Nous formulerons l'hypothèse qu'il témoigne d'un amenuisement de l'appartenance à une lignée familiale, mais aussi, dans une autre perspective, d'une séparation de la sexualité et de la procréation, entièrement avalisée à la fin des années 1970 avec la généralisation de la pilule contraceptive. En Afrique, par exemple, le rapport entre noms collectifs et appartenance n'est pas le même selon que les sociétés sont patri- ou matrilinéaires, ou qu'elles associent ou séparent sexualité et procréation (Pradelles de Latour, Charles-Henry 2000 et 2004).

\section{"Choisir" un nom de famille une approche psychologique du nom}

Appuyant ses propos sur ceux de Goethe, Freud soulignait en 1900 le lien étroit qui noue une personne à son nom : « on a grandi avec lui comme avec sa peau " (Freud 1967 : 184). Lorsqu'en effet le nom fait l'objet d'une distorsion, lorsqu'il est "ravalé au rang de nom commun " (Pradelles de Latour, Marie-Lorraine 1992:3) par accident ou encore par plaisanterie ou moquerie, les effets de signification dérangent souvent la personne. "Qu’il puisse faire sens n'est pas ce qui importe dans le nom propre. Tenir compte 
du nom propre comme nom propre consiste en ce rejet même, en ce traitement spécifique, qui ne le maintient comme nom propre quau prix de ne s'intéresser qu'à sa couleur ", note Jean Allouch (1984: 134). Dans le champ des sciences du langage comme dans celui de la psychologie, les auteurs s'accordent sur le statut particulier du nom propre dans la langue et dans la construction identitaire du sujet. "Nous savons ", écrit Roland Gori, «que les noms propres n'ont pas à signifier et qu'ils se distinguent des mots de la langue commune en ne renvoyant qu'à eux-mêmes. Faire signifier un nom, c'est commettre un sacrilège, transgresser un tabou, accomplir un sacrifice, lequel dénude le caractère insoutenable et évanescent des marques de notre identité comme de notre filiation» (1996: 140).

Cet attachement, Freud le rappelle en 1912 dans Totem et Tabou où, dit-il, le nom "fait corps» avec la personne, et en cela, poursuit-il, "la pratique psychanalytique concorde bien lorsqu'elle trouve de multiples occasions de renvoyer à la signification des noms dans l'activité de pensée inconsciente» $(1981: 263)$. Ce rapport intime, qui lie intégrité signifiante et intégrité de la personne, propulserait-il en quelque sorte le nom dans des rouages inconscients?

La réforme du nom sollicite en effet l'histoire de chaque personne dans une mobilisation qui peut se travailler selon deux points de vue : un point de vue qui prend en compte les liens du côté d'une inscription généalogique, reliant le sujet aux membres de son groupe familial - ascendants, descendants ou collatéraux - et définissant sa place comme assignée, en tant qu'être sexué dans la différence des générations; et un point de vue qui, se fondant sur l'hypothèse de l'inconscient, considère ces mêmes liens comme traces d' « expériences-vécues » (Pradelles-Monod 2001b) ou, pour reprendre les termes de Freud, "de choses vécues et de choses entendues » (1973: 181).

Comment les parents vont-ils s'approprier cette nouvelle donne juridique?

Avec les nouvelles conditions de son attribution, le nom n'est plus seulement le signe d'une appartenance à un groupe familial. Le simple fait qu'il puisse donner lieu à la possibilité d'un "choix ", même très cadré, lui confere une tout autre dimension que celle qu'il avait jusqu'à présent : il le colore de l'engagement subjectif de ceux qui auront retenu ce nom-là ou cette combinaison-là parmi les possibles. Les rationalisations de ce choix pourraient refléter la manière dont ces (en général jeunes) parents se construisent dans la différence des sexes et des générations notamment par ce qui les lie à leurs propres père et mère. Nous pouvons alors faire l'hypothèse que l'enfant pourrait être pris d'emblée dans la manière dont ce nom, pour ses parents, fait histoire, une histoire dont il deviendrait 
en quelque sorte le dépositaire. Selon la célèbre formule de Freud, les noms «font des enfants des revenants" (1967: 415), représentant parfois un lourd héritage. Qu'en est-il de cet héritage (Casper 2001) ? Le nouage du nom à l'histoire personnelle de chacun des parents et sa possible appropriation par l'enfant nous amène à situer la question dans le cadre de la transmission inter- et transgénérationnelle.

Dans un travail psychothérapeutique, il arrive que ce lien intime d'un sujet à ses nom(s) et prénom(s) surgisse et se déploie au sein d'une histoire familiale. C'est ainsi que dans le nom s'exprime une problématique psychique mettant en jeu la façon dont le sujet est lié aux personnes de sa famille, à ses frères et sœurs, à ses père et mère, à ses grands-pères et grands-mères, etc. Afin d'illustrer ce déploiement, nous retiendrons quelques éléments issus du suivi psychothérapeutique de Juliette ${ }^{13}$, qui témoignent de la façon dont le nom, pour elle, fait lien avec l'histoire de son père, celle de sa mère, de ses grands-parents et de son frère.

Juliette est une fillette de dix ans, suivie pour une forte inhibition scolaire. Inquiète pour cette enfant, la maîtresse avait en effet conseillé aux parents d'aller voir un psychologue. Juliette est décrite comme une enfant souvent en retrait, avec des résultats scolaires très irréguliers. Il lui est quasiment impossible de se projeter dans l'avenir, ce qui est interprété par son entourage scolaire comme une forme de refus de grandir.

À l'occasion de la mise en place du cadre psychothérapeutique, nous rencontrons Nathalie Behren, sa mère. Âgée de vingt-six ans, cette jeune femme est enfant unique de parents d'un certain âge qui ont longuement attendu et espéré la naissance d'un garçon. Exerçant comme esthéticienne, elle vit depuis plus de dix ans dans la ville où elle a rencontré celui qui est devenu son mari en 1992, Alain Cavaillès, ébéniste comme son père. On aurait envie d'écrire : "ébéniste-comme-son-père " tant l'un ne va pas sans l'autre. Travaillant dans une grande menuiserie et emmagasinant de l'expérience, Alain attend de succéder à un père qui, bien qu'ayant largement dépassé la soixantaine, ne semble pas encore prêt de lâcher l'entreprise familiale. Le souhait d'Alain de faire ajouter, dans un premier temps, au nom de l'atelier Cavaillès la mention père \& fils est toujours resté lettre morte.

Les parents de Nathalie sont très - trop - présents, "envahissants » même, dans une déception implicite et permanente de ce qu'elle est née fille. Nathalie conserve dans l'usage son nom de jeune fille sans pouvoir en expliciter les raisons.

En 1994, Nathalie met au monde Juliette et un an plus tard naît Adrien. Nathalie se retrouve mère de deux enfants très rapprochés par l'âge. Entre un patron exigeant et l'intendance domestique, elle rêve parfois d'une autre vie. Alain, de son côté, est comblé par l'arrivée de ce fils qui prolonge sa lignée dans la continuité du nom de famille. Comme le prévoit la loi, Juliette comme son frère Adrien porte le nom de son père, ce nom de Cavailless, qui lie le destin de la famille à celui de l'atelier d'ébénisterie, et qui semble être pour le père le trait d'une identité masculine, identité qu'il tient lui-même de son père et qu'il tend à son propre fils.

13. Il va de soi que nous avons respecté les règles éthiques de l'étude de cas en psychologie clinique en assurant l'anonymat des personnes, des lieux et des dates... 
Durant les séances, Juliette trace inlassablement les lettres de ce nom pour les associer successivement à son prénom puis à celui de son frère. Dans ce jeu d'écriture, le crayon de Juliette dessine une première fois le A d'Adrien, puis il passe sur le $\mathrm{A}$ encore une fois. Dans ce geste répété le A d'Adrien rencontre le A d'Alain son père et le A de Auguste son grand-père paternel. Juliette manifeste ainsi le lien étroit qui noue son nom de famille, Cavaillès, à son père. Sans que cela puisse se dire explicitement, ce nom patronymique, pour Juliette, ne représente pas le père dans sa fonction d'unificateur familial ; il le représente dans cette quête identitaire inlassable à vouloir hériter du père son entreprise familiale. Ce lien étroit, nous le retrouvons dans la parole de Nathalie sa mère, lorsque celle-ci nous dit combien elle est mal à l'aise, gênée par la manière dont son mari renvoie de façon récurrente chacun des enfants: Juliette aux Behren, Adrien aux Cavaillès. Dans un mouvement qui lui permet de séparer la lignée des femmes de celle des hommes, le père de Juliette fait jouer l'un par rapport à l'autre son nom et celui de sa femme.

Séance après séance, Juliette ne cesse de signifier combien elle se perd entre le souhait, impossible à réaliser, de faire partie des Cavaillès comme Adrien et la difficulté de faire partie des Behren, en résonance avec la féminité de sa mère dans ce qu'elle a de plus fragile, de moins assuré.

Ce que le travail psychothérapeutique fait surgir pour Juliette, c'est un nom qui ne peut plus soutenir pour elle une appartenance familiale parce qu'il fait exister ce frère qu'elle n'est pas et cette femme en mal de devenir. Dans les modalités de sa transmission et dans un effet de rencontre entre les fantasmes paternel et maternel, son nom pour Juliette vient dire une identification en souffrance.

Ce cas, comme bien d'autres, met l'accent sur les incidences psychologiques du nom et nous permet de formuler l'hypothèse que son choix pourrait activer en quelque sorte les sinuosités d'une histoire singulière qui se construit tant sur un versant conscient qu'inconscient. En effet, dans la réforme, le principe du choix introduit une forme de rivalité entre le nom du père et celui de la mère. Si Juliette était née en janvier 2005, comment le choix du nom se serait-il posé pour les parents de la fillette ? À quel nom auraient-ils été conduits? Nous pouvons penser que, quelle qu'ait été la décision des parents, ce choix aurait été infiltré par la problématique psychique mise au jour à l'occasion des difficultés de la fillette.

Plus largement, la question du nom et des changements portés par la nouvelle loi soulève celle de l'articulation entre les institutions sociales, notamment juridiques, et la personne dans sa construction subjective. L'illustration ci-dessus ouvre sur les représentations propres à chaque sujet dans son histoire et dans les relations particulières qu'il noue au sein de sa famille. Ces significations subjectives donnent au nom une dimension psychique qui fonctionne sur un mode autre que ceux à l'œuvre dans les domaines du social et du juridique. Cette dimension psychologique qui met en jeu des processus psychiques inconscients et dont nous ignorons le fonctionnement (sauf à l'interroger), implique que la maîtrise de nos actes, de nos paroles, de nos conduites nous échappe en partie : ainsi en est-il 
de nos choix, des plus anodins aux plus importants. Le choix du nom donné au premier enfant n'échappe pas à cette logique. Apparemment conduit par le raisonnement, il est en fait déterminé par des motivations qu'en chacun d'eux les parents ignorent. Ces motivations, qui se dérobent à toute forme explicative, mobilisent les parents dans leur histoire personnelle mais aussi dans les liens qui les unissent au sein du couple qu'ils forment. Davantage exposé aux «aléas " psychologiques, le nom, objet de choix, devient en quelque sorte l'attribut d'un père, l'attribut d'une mère.

L'économie psychique qui sous-tend ce mode de fonctionnement, nous amène à porter un autre regard sur le principe d'égalité qui se trouve au cœur de ce récent dispositif juridique. Sur le plan social, la disparition du patronyme y répond avec d'autres changements qui signent une évolution de la société occidentale. La démultiplication des échanges accélérant la sécularisation des biens et des valeurs inaliénables, tout être humain "échangeur ", homme ou femme, est mis sur un pied d'égalité. Les lois, qui s'adaptent aux changements sociaux, ont ainsi progressivement aboli les privilèges du mari, puis du père : suppression de son droit de punition corporelle sur les enfants (1895) et de sa puissance maritale sur son épouse (1938, 1942, 1965 et 1985), égalisation de sa responsabilité et de celle de sa femme (1970), et enfin suppression de la prédominance symbolique de son nom (2005). Le droit ne connaît désormais que l'égalité des conjoints. Enfin, la séparation progressive de la sexualité et de la procréation (Pradelles de Latour, Charles-Henry 2000, 2004) parachevée dans les années 1970 avec la généralisation de la pilule contraceptive, oblitère de plus en plus le rôle autrefois primordial accordé à la reproduction. Dans cette perspective, la disparition du patronyme répond au principe d'égalité. Signe du déclin de la représentation exclusive de la succession des générations par les pères, elle signale la réduction du groupe parental à une unité générationnelle, la fratrie. Le nom du père devenant l'égal du nom de la mère, le nom de famille choisi par les parents, qu'il soit composé ou non, devient, à l'instar du nom individuel, un nom «nouveau » donné à la naissance du premier enfant d'une fratrie.

Dès que nous passons de la loi à son application dans l'histoire d'une famille, nous passons de l'individu (considéré comme un parmi les autres) au sujet dans sa singularité. En d'autres termes, le principe d'égalité entre l'homme et la femme, entre le père et la mère, auquel répond la loi trouve en effet sa pertinence lorsque l'on considère l'homme et la femme comme des individus semblables, appartenant semblablement à la société qui les intègre. Mais qu'en est-il de sa pertinence si l'on se situe à l'échelle d'une famille, d'une histoire, des liens qui se créent entre des personnes, c'est-à-dire à une échelle subjective? Qu'en est-il de cette notion juridique d'égalité lorsque 
celle-ci est censée s'appliquer à des personnes prises dans des liens de couple extrêmement complexes? Lorsque, par exemple, le rapport à l'autre se vit dans l'aliénation, la soumission, la culpabilité, quelle est la portée de cette égalité ? Qu'en est-il de l'égalité dans une histoire où le parent est en dette inconsciente à l'égard d'un père, d'une mère, d'un grand-père, d'une grandmère... ? Le principe d'égalité qui vaut dans le domaine juridique parce qu'il ne connaît que l'individu, unité égale à toute autre, n'opère pas dans le domaine psychique. L'égalité est une notion que l'inconscient ignore.

Ces quelques développements nous permettent aujourd'hui de poser comme hypothèse que, en tant qu'il est l'objet d'un choix, le nom de famille prendrait valeur de nom individuel. L'écart entre nom individuel et nom collectif, fortement marqué comme nous l'avons vu dans certaines sociétés traditionnelles africaines, est dans notre société de plus en plus réduit. Dans cette évolution sociale où l'individualisme prend le pas sur tous les collectivismes, le groupe familial tend à se réduire à son unité minimale et à sa stricte fonction : unir entre eux des enfants de mêmes parents. Avec la notion de choix, de quelle «famille " le nom devient-t-il le représentant? Curieusement, c'est aujourd'hui que la loi introduit dans son vocabulaire l'expression si populaire de «nom de famille ", alors même que le choix de celui-ci entraîne une distinction nominative entre les parents et leurs enfants beaucoup plus marquée qu'elle ne l'était dans le système patronymique. Déjà en 1995, Pierre Murat constatait que notre législation ne connaissait "pas à proprement parler de "nom de famille" " tout en définissant strictement ce dernier comme "un nom commun à tous formé [lors de la constitution de la famille] à partir de l'un ou des deux noms des membres du couple" parental (1995: 218).

Dans le système patronymique, la valeur symbolique et sociale du nom tenait essentiellement à sa fonction de représentant d'une appartenance familiale au sens où il arrimait l'enfant aux deux lignées, paternelle et maternelle. Le patronyme représentait cette double filiation unie par des liens d'alliance, même s'il n'en indiquait pas concrètement les deux lignées : ses modalités de transmission étaient imposées par la loi (Casper 2005).

Dépendant alors des seuls liens légaux de filiation, c'était dans une totale extériorité que le nom était attribué à l'enfant. Reposant sur les seuls liens de filiation, tout à la fois il installait de la famille sur une origine et renvoyait à la famille d'où le sujet était issu. Le nom de famille, commun à tous les enfants reconnus d'un même couple, installe dorénavant de la famille sur la génération en train de se constituer. Soulignant le lien intragénérationnel, il renvoie davantage à la cellule familiale qui se crée. 
Le droit transfere au couple la responsabilité d'un choix (Murat 1995: 220) qui jusque-là incombait à la société. Certes, le système patronymique écartait le nom de la mère, mais la personne ne le décidait pas. Désormais le parent est responsable du choix du nom. Le nom devient entre l'enfant et lui un lien particulier forgé à partir d'une histoire éminemment singulière et contingente. Inextricablement lié à ce temps de la naissance, le nom choisi est marqué du sceau de l'événementiel.

\author{
Faculté de psychologie et des sciences de l'éducation \\ Université Louis Pasteur-Strasbourg I, Strasbourg \\ marie-claude.casper@psycho-ulp.u-strasbg.fr \\ Centre de droit privé fondamental \\ Université Robert Schuman-Strasbourg III, Strasbourg \\ frederique.granet@urs.u-strasbg.fr \\ Laboratoire d'anthropologie sociale, Paris \\ Centre national de la recherche scientifique \\ charles-henry.pradelles1@libertysurf.fr
}

MOTS CLÉS/ KEYWORDS : nom individuel/individual (first) name - nom collectif/collective (last) name - nom de famille/family name - génération/generation - nom (réforme française $\mathrm{du})$ /French law reforming the choice of a family name.

\title{
BIBLIOGRAPHIE
}

Allouch, Jean

1984 Lettre pour lettre : transcrire, traduire translittérer. Toulouse, Érès.

Casper, Marie-Claude

2001 «L'effet de transmission du prénom:

d'un héritage à son appropriation ", Cliniques méditerranéennes 64 (2) : 157-168. 2005 «La filiation à l'épreuve du choix : une approche clinique du nom de famille", Pratiques psychologiques 11:101-111.

\section{Dauzat, Albert}

1956 Les Noms de personnes : origine et évolution. Paris, Delagrave.

Fabian, Johannes,

1965 «!Kung Bushman Kinship :

Conponential Analysis and Alternative Interpretations ", Anthropos 60 : 663-718.

\section{Feschet, Valérie}

2004 "La transmission du nom de famille en Europe occidentale ", L'Homme 169: 6188.

Freud, Sigmund

1967 [1900] L'Interprétation des rêves. Paris, PUF.

1973 La Naissance de la psychanalyse. Paris, PUF.

1981 [1912] Totem et Tabou. Paris, Payot.

\section{Ganay, Solange}

1959 Les Devises des Dogons. Paris, Institut d'ethnologie ("Travaux et Mémoires de l'Institut d'ethnographie» 41).

\section{Gori, Roland}

1996 La Preuve par la parole: sur la causalité en psychanalyse. Paris, PUF. 
Houis, Maurice

1963 Les Noms individuels chez les Mosi.

Daker, s.n. - Limoges, Impr. A. Bontemps.

Kripke, Saul A.

1982 La Logique des noms propres. Paris, Minuit.

\section{Lamizet, Bernard}

2001 "L'égalité, l'identité et le nom », Le Monde, 3 février.

Lapierre, Nicole

1995 Changer de nom. Paris, Stock.

Lebel, Paul

1981 Les Noms de personnes en France. Paris, PUF.

Lefebvre-Teillard, Anne

1990 Le Nom : droit et histoire. Paris, PUF.

Legendre, Pierre

1985 L'inestimable objet de la transmission: étude sur le principe généalogique en

Occident. Paris, Fayard ("Leçons» 4).

Legendre, Pierre

\& Alexandra Papageorgiou-Legendre

1990 Filiation: fondement généalogique de la psychanalyse. Paris, Fayard ("Leçons" 4, suite 2).

\section{Lifchitz O. \& Denise Paulme}

1952 "Les noms individuels chez les Dogon ", in Mélanges ethnologiques. Dakar, IFAN (" Mémoires de l'Institut français d'Afrique noire » 23 ) : 307-357.

Martin, Jean-Luc

1995 "Nom et loi ", in Lucette Khaïat, ed., Vérité scientifique, vérité psychique et droit de la filiation. Toulouse, Érès : 228-238.
Middleton, John

1957 "The Social Significance of the Lugbara Personal Names », The Uganda Journal $21: 34-42$.

\section{Murat, Pierre}

1995 «Le nom de l'enfant : plaidoyer pour un statu quo ", in Lucette Khaïat, ed., Vérité scientifique, vérité psychique et droit de la filiation. Toulouse, Érès : 217-227.

\section{Pradelles de Latour, Charles-Henry}

2000 «Quand la sexualité et la procréation sont séparées ", Cliniques méditerranéennes 63 (1) : 89-101.

2004 "Changements de sexualité, changements de croyances et de paternités ", L'Unebévue 22 : 207-219.

\section{Pradelles de Latour, Marie-Lorraine}

1984 "Double filiation et identités", Littoral 11-12: Du père: 48-53.

1992 «Entre le dire et l'écrit... Les frontières du nom ", Tradisis : revue du LadisisProparlan $0: 1-9$.

1996 "Une origine falsifiée ", L'Évolution psychiatrique 61 (2) : 465-470.

1997 "Transmissions généalogiques: de l'événement à la trace de l'événement ", Revue internationale de psychopathologie 24 : 33-46.

\section{Pradelles-Monod, Marie-Lorraine}

2001a "Sous la filiation : l'ancestralisation?", Cliniques méditerranéennes 63 (1) : 31-37.

2001b "Les "constructions filiatives" : une formation de compromis? (Pour une clinique du lien de filiation) ", L'Évolution psychiatrique 66 (1) : 105-110. 
Marie-Claude Casper, Frédérique Granet \& Charles-Henry Pradelles de Latour, Choisir un nom de famille... Une approche pluridisciplinaire des implications de la réforme du nom.Juristes, psychologues et anthropologue, réunis dans un groupe de recherche pluridisciplinaire, ont joint ici leurs réflexions pour cerner les enjeux de la nouvelle législation portant sur le nom de famille, introduite en France en janvier 2005. Le fait que ce nouveau nom appelé «nom de famille» puisse faire l'objet d'un choix à chaque génération, par les conjoints qui fondent une nouvelle cellule familiale, indique que l'écart existant entre nom individuel (prénom) et nom collectif (patronyme) va se réduire. Elle souligne aussi que le groupe parental, qui rassemblait sur plusieurs générations les descendants agnatiques d'un ancêtre commun, pourra être par le choix du double nom (côtés père et mère) ramené à un nom de fratrie. L'article montre que cette nouvelle donne a été largement induite par l'abolition des privilèges réservés à la fonction paternelle et par la séparation progressive de la sexualité et de la procréation qui efface le rôle primordial attribué jusqu'ici à la reproduction. Mais comment cette égalité des sexes avalisée dans le droit familial résonne-t-elle dans l'inconscient?
Marie-Claude Casper, Frédérique Granet \& Charles-Henry Pradelles de Latour, Choosing a Family Name:A Multidisciplinary Approach for Studying the Implications of the Reform.Jurists, psychologists and anthropologist meeting in a multidisciplinary research group have shared their ideas about the issues raised by the January 2005 act of French law reforming the choice of a family name. The fact that this new so-called family name can be chosen in each generation by the spouses who found a family unit is evidence that there will be less distance separating the individuals first and collective (patronymic) last names. The parental group, which used to refer to the agnatic descendants down through several generations of a common ancestor, might tend to become (owing to the choice of a name combining both the farthers and mothers last names) the name of a sibling group. This new situation tends to emerge both from the abolition of the privileges previously reserved for the paternal function and from a gradual separation of sexuality and procreation, which erases the primordial role that reproduction used to have. But what echoes does this equality of the sexes, as established in family law, have in the subconscious? 